\title{
Integrated Medical Care
}

\section{Ausgangssituation}

Das deutsche Gesundheitswesen ist durch eine mangelnde Integration der Versorgungsabläufe gekennzeichnet - speziell bei Patienten mit komplexen Behandlungsabläufen und chronischen Erkrankungen. Zurückzuführen ist dies insbesondere auf die streng sektorale und damit höchst artifizielle Trennung von ambulanter und stationärer Versorgung. Eine zusätzliche Barriere für die Realisierung kontinuierlicher Diagnostik- und Behandlungsabläufe bildet die historisch begründete örtliche und budgetäre Trennung von Akut- und Rehabilitationsmedizin [4]. Zudem werden Rehabilitationsleistungen noch überwiegend in meist wohnortfernen, stationären Einrichtungen angeboten. Auch die rigide Vertrags- und Vergütungsstruktur für die Versorgung von Patienten mit Arzneimitteln und anderen Heil- und Hilfsmitteln steht den ökonomischen Anforderungen entgegen, die eine integrierte Versorgung ohne zeitliche und inhaltliche Brüche ermöglichen sollen [4].

Strikt getrennte Behandlungssektoren orientieren sich selten am konkreten Versorgungsproblem. Sie verursachen eine ausgabenintensive Doppelversorgung mit Personal (z.B. ambulante und stationäre Facharztstruktur), mit Sachmitteln und Investitionsgütern (z.B. Großgeräte in Krankenhaus und Praxis). Auch besteht ein vermehrter Raumbedarf. Sektorale Budgetvorgaben und Wirtschaftlichkeitsprüfungen in den einzelnen Versorgungsebenen induzieren außerdem kräftige ökonomische Anreize für die Entwicklung von Auslastungsstrategien, die einen medizinisch sinnvollen Einsatz der immer knapper werdenden Ressourcen verhindern. Das oft langjährige Einzelkämpfertum und insbesondere die im Zusammenhang mit komplexen Erkrankungen zu geringe Erfahrung und zu niedrige Untersuchungsfrequenz wirken sich nachteilig auf die Effizienz und Qualität der fachärztlichen Vorsorgung aus [1,4].

\section{Neue Versorgungsformen}

Grundsätzliches Anliegen der neuen Gesundheitsreformgesetze, die ab 1.1. 2004 gelten, ist die Etablierung potenziell sektorübergreifender Versorgungsstrukturen mit Sicherstellung einer effizienten und qualitätsgesicherten Behandlung aus einer Hand [2]. Der Gesetzgeber hat damit die rechtlichen Rahmenbedingungen für neue Versorgungsangebote geschaffen, die eine Flexibilisierung von Versorgungsstrukturen gestatten, Schnittstellenprobleme minimieren und kontinuierliche Behandlungsabläufe und -pläne ermöglichen. Damit werden Anreize gesetzt, die zu mehr Wettbewerb, Gestaltungsspielraum, Wirtschaftlichkeit und Qualität führen sollen - aber auch zu völlig neuen Organisationsstrukturen, Beteiligungskonzepten und einer Intensivierung der Fort- und Weiterbildung. Im Kern hat der Gesetzgeber die Rahmenbedingungen für folgende Versorgungsformen definiert [2-4]:

- Hausarztzentrierte Versorgung ( $§ 73 b$ SGB V)

- Förderung der Qualität in der ärztlichen Versorgung (§73c SGB V)

- Medizinische Versorgungszentren (§95 SGB V)

- Teilöffnung der Krankenhäuser ( $§ 116 b$ SGB V)

- Integrierte Versorgung ( $\$ 140 a-d$ SGB V) 
Ziele und Rahmenbedingungen der Integrierten Versorgung

Der Gesetzgeber versteht unter der Integrierten Versorgung (IV) eine übergreifende Versorgungsstruktur mit verschiedenen Leistungssektoren (z.B. ambulant, stationär, poststationär), die zusätzlich oder alternativ eine interdisziplinär übergreifende Versorgung (z. B. Hausarzt, Kardiologe, Pneumologe) beinhalten [2] Im Vordergrund steht dabei die standardisierte Versorgung vornehmlich chronisch Kranker mit mehr Qualität, verbesserten Abläufen, weniger Schnittstellen und geringeren Kosten. Die Einbindung der IV in den Sicherstellungsauftrag der Kassenärztlichen Vereinigung entfällt. Dadurch bietet sich Krankenhäusern die Chance der Weiterentwicklung von einer ausschließlich stationär handelnden Einrichtung zu einem medizinischen Dienstleistungszentrum mit übergreifendem Versorgungsangebot [1,4].

\section{Verträge zur IV}

Ein Schwerpunkt der IV ist, Schnittstellenprobleme zu überwinden und die Organisation sektorübergreifend sicherzustellen. Ferner soll die Beteiligung im Hinblick auf Leistungen, qualitativ hochwertige und wirtschaftliche Erbringung und optimale Inanspruchnahme unterstützt werden [5]. Um die Versorgung aus einer Hand zu gewährleisten, ermöglicht die IV die Schließung von Einzelverträgen zwischen Kostenträgeren (z.B. eine oder mehrere Krankenkassen gemeinsam) und Leistungserbringern (z.B. Vertragsärzte, Praxisgemeinschaften, Medizinische Versorgungszentren, Krankenhäuser, Physiotherapeuten) sowie Managementgesellschaften (z.B. für die Fallsteuerung, Koordination, Dokumentation, Evaluation, Abrechnung) als Auftragnehmer der Kostenträger oder Auftraggeber der Leistungserbringer. Ebenfalls neu ist, dass Leistungserbringer auch außerhalb des regulären Zulassungs- oder Ermächtigungsverfahrens über Direktverträge an der IV partizipieren können. Vertragsgegenstand dürfen nur solche Leistungen sein, über deren Eignung der gemeinsame Bundesausschuss keine ablehnende Entscheidung getroffen hat. Nach Abschluss eines Vertrages zur IV besteht Gewährleistungspflicht für die Patientenversorgung durch die Vertragspartner. Diese beinhaltet auch die gebotene Qualität, Zweckmäßigkeit und Wirtschaftlichkeit. Qualitäts- und Wirtschaftlichkeitsvorteile der IV gegenüber der Regelversorgung müssen konkret definiert und nachvollziehbar kalkuliert sein. Steuerung, Dokumentation und Evaluation müssen auf der Basis vorstrukturierter Mindeststandards geregelt sein. Jeder Vertrag beinhaltet mindestens ein medizinisches und ein ökonomisches Modell, das den Status quo des Versorgungsproblems mit den Möglichkeiten der „neuen“ Integrationsversorgung in Beziehung setzt [4]. Alle IV-Verträge werden zentral registriert. Als gemeinsame Registrierstelle fungiert die Bundesgeschäftstelle Qualitätssicherung $\mathrm{GmbH}(\mathrm{BQS})$ in Düsseldorf (www.bgs-register 140d.de), die auf Anfrage über registrierte Anträge Auskunft erteilt.

\section{Freie Arztwahl und IV}

Die Teilnahme des Versicherten an IV-Programmen ist freiwillig. Hat der Patient sich jedoch einmal eingeschrieben, darf er nur die vorgegebenen Leistungserbringer in Anspruch nehmen. Wer für die Einschreibung verantwortlich ist, wird im IV-Vertrag klar geregelt. Anreize zur Einschreibung bieten Boni für gesundheitsbewusstes Verhalten, Zuzahlungs- und Beitragsrabatte, wie sie von den privaten Krankenversicherungen bekannt sind.

\section{Anschubfinanzierung, Wettbewerbsrecht und IV}

Die Anschubfinanzierung der IV erfolgt durch Rechnungskürzung. Jede Krankenkasse hat das Recht, die Gesamtvergütung für die Kassenärztliche Vereinigung und die stationären und teilstationären Krankenhausrechnungen von Anfang 2004 bis Ende 2006 um maximal 1\% zu kürzen, sofern diese Mittel tatsächlich zur Umsetzung der IV benötigt werden. Die einbehaltenen Mittel werden für Leistungserbringer extrabudgetär zur Verfügung gestellt, die sich an der IV beteiligen. IV-Verträge sind als Konzessionsverträge - zumindest nach Auffassung der GKV - vom Vergaberecht ausgenommen. Sie fallen somit nicht unter das Gesetz gegen die Wettbewerbsbeschränkung (GWB). Wenn die IV-Verträge dagegen nicht nur den Preis (Vergütungshöhe) garantieren, sondern auch bestimmte Mengen (Fallzahl), unterliegen sie als öffentliche Aufträge dem GWB und müssen bei Überschreitung definierter Schwellenwerte ausgeschrieben werden.

\section{Risiken und Chancen der IV fürr die Pneumologie}

\section{Welche Krankheitsbilder sind geeignet?}

Grundsätzlich werden Modelle zur IV nur dann erfolgreich sein, wenn diese sich für alle (Patient, Krankenhaus, Arztpraxis, sonstige Anbieter, Krankenkasse) lohnen. Zwar würde eine populationsbezogene IV stärkere Impulse für eine gesundheitsökonomisch sinnvolle und zielgerichtete Umgestaltung der existierenden Versorgungsstrukturen setzen als indikationsbezogene Integrationsmodelle, doch ist diese nur schrittweise und mit wesentlich größeren Risiken für Leistungsanbieter und Krankenkassen umsetzbar. Soll das Konzept der IV bereits mittelfristig zum Erfolg führen, stehen indikationsbezogene IV-Modelle ganz im Vordergrund. Aus Sicht der Pneumologie bieten sich IV-Modelle hauptsächlich für folgende Krankheitsbilder an:

- Chronisch obstruktive Atemwegserkrankungen (COPD, Asthma bronchiale)

- Bronchialkarzinom (Lungenkrebs)

- Schlafapnoe

- Mukoviszidose

- Lungentransplantation

- Respiratorische Insuffizienz

\section{Chancen der pneumologischen Klinilk}

Die stärkste medizinische Stellung und das höchste Vertrauen der Patienten in die lokalen Versorgungsstrukturen haben zweifellos die Kliniken. Das Modell der IV setzt keine Anreize zum weiteren Ausbau der flächendeckenden fachärztlichen Versorgung in Einzelpraxen, sondern motiviert zur Etablierung kreativer Betreuungskonzepte in Kooperationsverbünden mit Krankenhäusern. Die Ausgangssituation der pneumologischen Klinik ist schon deshalb günstig, weil das Zentrum der IV gewöhnlich dort liegt, wo für die potenziell intensivste medizinische Inter- 
vention die Vorhaltung der personellen, funktionalen und technisch-apparativen Infrastruktur unabdingbar ist. Die medizinische und betriebswirtschaftliche Infrastruktur erlaubt es der pneumologischen Klinik, mit leistungsfähigen und kompetenten Kooperationspartnern durchgängige Versorgungskonzepte zu gestalten, diese zu koordinieren und erfolgreich umzusetzen. Das gesetzliche Regelwerk der IV gestattet ferner die Einbeziehung von Leistungsanbietern in DMP-Programmen und von medizinischen Versorgungszentren sowie die Beteiligung von Dritten (z. B. für Arznei-, Heil- und Hilfsmittel). Auch diese Form der Integration dient eindeutig dem Ziel der übergreifenden Steuerung des Versorgungsauftrages im Rahmen der IV und eröffnet pneumologischen Kliniken (Abteilungen) damit zusätzliche Perspektiven. Durch die arbeitsteilige Vernetzung resultiert für die koordinierende Klinik zwar nicht selten eine Fallzahlreduktion bei leichten Erkrankungen, doch nimmt die Zahl und Intensität der schweren Fälle anteilig zu. Das Krankenhaus muss diese Leistungsverdichtung pro Patient zukünftig nicht fürchten, da im DRG-System ein dem Schweregrad angepasster Erlös erzielt wird. Durch schrittweise Implementierung von Leitlinien zur Steuerung der Diagnose- und Behandlungsabläufe kann die Erlössituation überdies entscheidend verbessert werden [1-4]. Alle Initiativen erfordern Management. Auch hier sind Krankenhäuser regional am ehesten in der Lage, Kapazitäten zu mobilisieren [5].

\section{Chancen für pneumologische Praxen}

Die Beteiligung an Modellen der IV bietet dem niedergelassenen Facharztes für Lungen- und Bronchialheilkunde und dem spezialisierten Pneumologen (Schlafmedizin, Allergologie) attraktive Gestaltungsspielräume, die über die Hoffnung auf Rückgewinnung verlorener Budgetanteile durch Beteiligung an integrierten Leistungsangeboten hinausgehen. Durch Übernahme konkreter Verantwortlichkeiten und Mitgestaltung der zweckmäßigsten Abläufe und Mindestanforderungen an die Qualität wird das Schnittstellenmanagement zwischen ambulantem und stationärem Sektor nachhaltig verbessert [1]. Dank vertraglich geregelter Kooperation mit dem Krankenhaus kann der niedergelassene Pneumologe die vor- und nachgelagerte Versorgung stationärer Patienten übernehmen, so dass sich Leistungen innerhalb und außerhalb des Krankenhauses bestmöglich ergänzen. In Versorgungspfaden ist vorab zu klären, welche zusätzlich vergüteten Aufträge der niedergelassene Facharzt unter welchen Rahmenbedingungen übernimmt. Infrage kommen zahlreiche auf Krankheitsbild und Stadium der Erkrankung abgestimmte Maßnahmen in der Prävention, Diagnostik, Therapie, Rehabilitation und Schulung der eingeschriebenen Patienten. Diese Maßnahmen sind mit einer leistungs- und qualitätsdefinierten extrabudgetären, auf medizinische Endpunkte bezogenen Vergütung gekoppelt. Je nach Krankheitsbild und Definition im IV-Vertrag übernimmt der niedergelassene Arzt des Weiteren die Einschreibung der Patienten und ist damit maßgeblich an der Mengensteuerung beteiligt. Ein nicht zu unterschätzender ökonomischer Anreiz der IV für niedergelassene Pneumologen ergibt sich aus der vertraglich geregelten Einbindung in intensive Kooperationsverbünde mit anderen spezialisierten Einzelpraxen und Praxisnetzen und der vertraglich geregelten Nutzung von Räumlichkeiten und Einrichtungen des Krankenhauses. Schließlich eröffnet die IV allen Beteiligten die Chance zur strukturierten Fort- und Weiterbildung in einem Ärzteteam mit professionellem Manage- ment, das im Idealfall Behandlungsprozesse etabliert und weiterentwickelt, die evidenzbasiert und leitlinienorientiert sind.

\section{Chancen aus der Sicht von DGP und BdP}

Zwar hängt der Erfolg der IV maßgeblich vom Motivationsgrad und dem Willen zur Zusammenarbeit der regional kooperierenden Leistungserbringer ab. Eine gemeinsame Kommission von Deutscher Gesellschaft für Pneumologie und Bundesverband der Pneumologen könnte jedoch überregionale Impulse zur Gestaltung von IV-Verträgen geben, Musterverträge bereitstellen und fach- und sachkompetente Beratungsmöglichkeiten organisieren.

\section{Risiken der IV}

Reale Gefahren in der erfolgreichen Umsetzung integrierter Versorgungsleistungen durch Überwindung hinderlicher Sektorengrenzen und Ausbildung bedarfsgerechter, zukunftsorientierter Angebotsstrukturen bestehen sowohl für Leistungsanbieter wie Kostenträger. Risiken ergeben sich unter anderem dadurch, dass sich Leistungen innerhalb und außerhalb des Krankenhauses breit überlappen, anstatt sich zu ergänzen. Ohne Zweifel sind alle IV-Programme gefährdet, deren kostenneutrale Fortsetzung nach Ablauf der Anschubfinanzierung Ende 2006 unrealistisch erscheint. Aus Sicht der Pneumologen als Anbieter integrierter Versorgungsleistungen besteht ein nicht zu unterschätzendes Risiko darin, dass sich die Unterdeckung eines Programms erst langfristig abzeichnen könnte. Gründe dafür sind eine fehlerhafte Einschätzung der Teilnehmerzahl, der Risikostruktur und der Morbiditätsentwicklung des Kollektivs oder die Vereinbarung unrealistischer Gewährleistungen und Boni. Auch die bislang vom Gesetzgeber nicht ausreichend definierten Anforderungen und Bewertungskriterien für Modelle der IV stellen für alle Akteure ein nicht zu unterschätzendes Gefährdungspotenzial dar. So sind beispielsweise die rechtlichen und organisatorischen Anforderungen an das interne und externe Controlling und die Art und Umsetzung von geeigneten Sanktionsmechanismen weitgehend unklar. Werden IV-Verträge mit kleinen oder nur regional operierenden Krankenkassen abgeschlossen, besitzen diese Konzepte im Regelfall keine das Gesundheitssystem dauerhaft verändernde Gestaltungskraft. Die größte Gefahr für die Pneumologie besteht aber ohne Zweifel darin, dass wir nicht konzertiert versuchen, flächendeckend zukunftsweisende IV-Modelle zu verankern. Diese garantieren nämlich sektorenübergreifend eine effiziente, kostengünstige und qualitätsgesicherte Versorgung von Patienten mit pneumologischen Erkrankungen, die entweder aufgrund der Epidemiologie oder ihres komplexen Charakters bestens dazu geeignet sind.

\section{Literatulr}

${ }^{1}$ Bauer A. Die Integrierte Versorgung kommt - aber wie? In: McKinsey Health. 2004; $1: 30$

2 GKV-Modernisierungsgesetz 2003; Gesetz vom 22.12.2003 - BGB I Seite 2626, insbesondere Abschnitt $11 \S \S 140$ a bis 140d SGB V.

${ }^{3}$ Lehnert H, Meyer J, Riemann J et al. Konzept der integrierten Versorgung und deren Bedeutung für die Innere Medizin. Der Internist 2004; 45: M239

${ }^{4}$ Rebscher H. Integrierte Versorgung. Arzt und Krankenhaus 2004; 4 : 113

${ }^{5}$ Schönbach KH. Verbesserte Bedingungen für die integrierte Versorgung. Die BKK 12/2003 